\title{
Los postulados biotipológicos en el curso para profesores de educación física - CNEF - Uruguay (1948-1956)
}

\author{
The biotipological postulates in the course for physical education \\ teachers - CNEF - Uruguay (1948-1956)
}

\author{
Camilo Rodriguez Antúnez \\ Orcid: https://orcid.org/0000-0001-8305-4335 \\ Instituto Superior de Educación Física, Universidad de la República, Montevidéu, \\ Uruguay, camilo.rodriguez.ur206@gmail.com
}

Received on 30/05/2020 - Approved on 05/06/2020

\begin{abstract}
Resúmen
El presente trabajo forma parte de una investigación1 relacionada al desarrollo de la eugenesia en Uruguay y sus vinculaciones con la educación física durante el periodo 1920-1948. Específicamente en este artículo, nos detendremos a visibilizar y analizar las formas en las que educación física y la biotipología se articularon en torno a la formación de profesores de educación física en el Uruguay. Buscaremos dar cuenta de las continuidades discursivas establecidas entre eugenesia, biotipología y educación física, así como las principales implicancias que se desprenden de estos vínculos para pensar la educación del cuerpo. La metodología parte del análisis histórico de fuentes primarias, con particular énfasis en el análisis del discurso a partir de las premisas brindadas por Foucault en relación a la "metodología arqueológica".
\end{abstract}

Palabras clave: Cuerpo. Eugenesia. Biotipología. Educación Física.

\begin{abstract}
This work is part of an investigation related to the development of eugenics in Uruguay and its links with physical education during the period of 1920-1948. Specifically in this article, we will focus on making visible and analysing the ways in which physical education and biotipology were articulated around the training of physical education teachers in Uruguay. We will seek to account for the discursive continuities established between eugenics, biotipology and physical education, as well as the main implications that emerge from these links to think about the education of the body. The methodology starts from the historical analysis of primary sources, with particular emphasis on the analysis of the discourse from the premises provided by Foucault in relation to the "archaeological methodology".
\end{abstract}

Keywords: Body. Eugenics. Biotipology. Physical education. 


\section{Introducción}

La biotipología fue una disciplina creada por el médico italiano Nicola Pende (18801970) en 1922. Este último nació en la ciudad italiana e Bari en 1880 y en 1903 obtuvo el título como médico por la Universidad de Roma a partir de su trabajo final vinculado a la "inervación de la glándula suprarenal" (Galera, 2005). Dentro de la formación de Pende aparecen referencias a la escuela constitucionalista de Giacinto Viola, de la neoconstitucionalista de Achille De Giovanni — preocupada entre otras cosas por integrar religión y ciencia - y a los postulados de la escuela criminológica de Cesar Lombroso. El abordaje de Pende se centraba en indagar sobre las vinculaciones entre el comportamiento y el sistema endocrino humano, obteniendo como parte de este proceso la formulación de su teoría cuyo nombre acuñó en su libro de $1922^{2}$. Dentro de este trabajo Pende definía a la biotipología como la "ciencia de los biotipos humanos somáticos y psíquicos” (Pende, 1939, apud Galera, 2005, p. 369). Su objetivo pasaba por identificar las cualidades biotípicas de cada individuo, es decir, dejar de pensar al hombre como un ser abstracto, igual a cualquier otro, para pasar a identificar cuáles eran las cualidades biológicas que diferencian a un hombre de otro y de esas cualidades qué efectos surtían sobre la psique y la conducta. La constitución individual, el biotipo, se entendía que se encontraba vinculado a las características morfológicas, fisiológicas y psicológicas. Desde la perspectiva del autor, la variable corporal, el resultado de esas tres dimensiones, era brindado en mayor medida por las leyes de la herencia biológicas que por los efectos ambientales (Galera, 2005, p. 370).

La figura de Pende y sus postulados fueron reconocidos mundialmente, proliferando instituciones afines a su teoría en una gran cantidad de países donde Uruguay no fue la excepción. Entendemos que parte de este reconocimiento se debe al desarrollo de los postulados biotipológicos en el marco del régimen fascista. Nicola Pende fue una figura importante dentro del Partido Nacional Fascista, llegando a ser senador por ese partido en 1933. En 1925 inauguró como rector la "Universidad Adriática Benito Mussolini", en su ciudad natal de Bari. Los lazos establecidos con el régimen le posibilitaron la concreción de un conjunto de instituciones, tanto en Italia como en diferentes países de Europa y América del Sur (donde se destaca Argentina), para el desarrollo de sus indagaciones y la aplicación política de sus ideas vinculadas a fortalecer a la "raza mediterránea". Para la justificación y consolidación de su proyecto de unidad biológico-moral, el fascismo contó con los insumos científicos y políticos producidos en el marco de la teoría de Nicola Pende. La biotipología fue una de las forma sobresaliente que adopto la eugenesia ${ }^{3}$ italiana y, a su vez, en estrecho vínculo con este desarrollo y a partir de la década de 1930, también en Argentina (Miranda, y Vallejo, 2012) y Uruguay (Sapriza, 2001) fue el modelo predominante del discurso eugenésico.

La biotipología, como "ciencia" que posibilitaba el acceso y la determinación de las cualidades corporales y mentales de los hombres, les proporcionaba a los estadistas información de calidad para organizar la sociedad en función de la biología y optimizar las aptitudes corporales de cada individuo en función de las lógicas del "organisismo social". En términos de Vallejo (2005, p. 248), una de las primeras leyes de la biotipología, y que a su vez fue una de las ideas claves que se articularon en torno a las políticas del fascismo, refiere a la idea del "althruismo celular". Desde esta perspectiva todas las unidades celulares de la sociedad, siendo la más pequeña el ser humano, debían colaborar con el resto de las unidades, propiciando que el gran "organismo social" funcione. El "altruismo" 
se encontraba vinculado a la renuncia de las libertades individuales en función del orden orgánico colectivo, a la necesidad de que cada uno ocupe el "justo lugar" que le corresponde biológicamente para que el gran "organismo social" funcione.

En ocasión de los postulados de Pende y en clara coordinación política con el autor italiano, en Argentina en 1932 se creo una de las instituciones más importantes de América del Sur vinculadas a la biotipología y la eugenesia como fue la Asociación Argentina de Biotipología Eugenesia y Medicina Social. Esta institución fue de vital importancia en la consolidación de las redes eugenésicas en el Uruguay y en la producción del discurso eugenésico en la región. A su vez, la misma contó con una área vinculada a la educación física a partir de la cual se pueden visualizar algunas de las articulaciones que se realizaron entre biotipología, eugenesia y educación física (Reggiani, 2014).

La educación física formó parte de las preocupaciones biotipológicas de Pende en la medida que se constituía como una fuerte herramienta para impulsar la formación física, moral e intelectual de la población orientada desde la perspectiva de la "biología política" (Vallejo, 2012). Si bien el discurso de Pende mantenía fuertes continuidades discursivas con el "decadentismo" (Reggiani, 2014), visualizando a los factores urbanosindustriales como los responsables de la decadencia racial, al pensar al hombre desde la biotipología y en articulación con la educación física lo hacía en función del esquema de la máquina: "desde el punto de vista energético, el organismo humano puede ser considerado como una máquina perfecta [...] máquina cuyo rendimiento está asegurado por tres motores: el motor muscular, el motor intelectual y el motor sentimental" (Pende, 1933, p. 9).

La biotipologia, como ciencia de la individualidad, debía aportar insumos de calidad sobre las tres dimensiones de la máquina para determinar las características de la misma y orientar su funcionamiento acorde a sus cualidades y a la eficiencia del "organismo social". La educación física, si bien era una práctica centrada en la maquinaria muscular, también lo era de la "voluntad, del sentimiento y del intelecto" (Pende, 1933, p. 9). Siguiendo los postulados de Jacinto Viola, Pende sostenía dentro de su teoría la existencia de cuatro tipologías sobre la morfología corporal de los hombres:

el tipo de línea larga pero funcionalmente fuerte (longilíneo sténico), el tipo de líneas largas pero funcionalmente débil (longilíneo sténico), el tipo de líneas largas pero funcionalmente débil (longilíneo asténico), el tipo de líneas breves y groseras, pero funcionalmente fuerte (brevilíneo sténico) y el tipo de líneas breves pero funcionalmente debil (brevilíneo asténico)". (Pende, 1933, pp. 9-10).

A partir de esta base, que a su vez posibilitaba pensar desde la perspectiva del autor en los tipos de inteligencia y carácter acorde a las cualidades tipológicas, era que se debía realizar los estudios y fichas biotipológicas para determinar las características de los individuos, seleccionarlos y dirigirlos acorde a sus cualidades. Los educadores de lo "físico psiquico" debían orientar sus prácticas de forma racional y tomando como referencia a los antecedentes de la personalidad "psicofísica" de los individuos, es decir: su "biotipo". Los exámenes, las fichas, servían para identificar las "cualidades musculares individuales", las cuales eran "un reflejo claro y muy instructivo tanto del estilo corpóreo individual como del temperamento y del tipo intelectivo del sujeto" (Pende, 1933, p. 10). 
De estos enunciados se desprende explícitamente la idea de que había sujetos cuya inteligencia y temperamento era funcionalmente débil-lenta y aquellos que no.

La lógica de jerarquización de los individuos a partir de su biología debía determinar, sobre todo en algunos espacios específicos del Estado fascista, la selección y generación de la "aristocracia biológica" proclamada por Pende. Se estipulaba que dentro de esta aristocracia biológica ingresarían los deportistas (Teja, 2002) a los cuales se seleccionaba por medio de los estudios biotipológicos: "sobre todo persiguiéndose el propósito de tener una orientación para efectuar una primera selección de sujetos" (Pende, 1933, p.11). En la línea de Pende, dichos exámenes, de vital importancia para la educación física y el adiestramiento atlético, debían ser realizados por personal especializado en biotipología y en instituciones afines.

A partir de la década de 1930 en América del Sur, tal como había sucedido en Italia, los estadistas comenzaron a realizar articulaciones entre biotipología y educación física. Como hemos mencionado, la Asociación Argentina de Biotipología Eugenesia y Medicina Social contó con una sección vinculada a la educación física de la cual formó parte, entre otros, Enrique C. Romero Brest (1873-1958) ${ }^{4}$. Desde el órgano de difusión de la Asociación denominado "Anales" se realizaron importantes publicaciones que vinculaban a la eugenesia, biotipologia y educación física. A su vez, desde la Asociación se creó una formación en biotipología donde la educación física formó parte de las asignaturas que se dictaban. Galak (2016), quien ha indagado en torno a cómo los discursos vinculados al higienismo, la eugenesia, la raza y la cultura física han influido en la educación del cuerpo en la región, sostiene que tanto en Brasil como en Argentina, la conjunción entre postulados eugenésicos, biotipológicos y psicologicistas tuvieron una gran influencia en el contexto educativo y que ese ideario generó importantes afectaciones para pensar las prácticas de juegos, gimnasias, deportes, etc. A su vez, Dos Santos (2012), sostiene que los supuestos teóricos- metodológicos de la biotipología tuvieron una incidencia considerable en la conformación del campo de la educación física brasilera, ingresando como una disciplina dentro de la formación de profesores de educación física. Como buscaremos desarrollar en el próximo apartado, los referentes de la educación física en Uruguay no fueron ajenos a la afectación de estos discursos que vinculaban educación física con biotipología, generando importantes políticas vinculadas a la educación del cuerpo en el país.

\section{Los postulados biotipológicos en ocasión de la formación de profesores de educación física}

A partir del "plan de estudio" de 1948, ingresó a la formación de profesores de educación física ${ }^{5}$ de la Comisión Nacional de Educación Física- Uruguay (CNEF) la asignatura biotipología. Esta incorporación, desde nuestra perspectiva, representa un hito clave para pensar los vínculos entre los postulados de la eugenesia y la educación física en el Uruguay ya que, como buscaremos desarrollar a lo largo de este capítulo, la biotipología se convirtió en una de las principales auxiliares de la tarea eugénica. En este capítulo nos centraremos en analizar algunas de las principales implicancias de esta incorporación. 


\section{Antecedentes}

Dentro del proceso de indagación documental nos hemos encontrados con algunas referencias específicas que, de forma incipiente, nos permiten sostener que los postulados biotipológicos se encontraban circulando dentro del campo de la educación física en el Uruguay previamente a la incorporación del curso de biotipología en 1948. Particularmente, identificamos las referencias que aparecen en relación a la prueba de ingreso ${ }^{6}$ al Curso de Profesores en 1939 donde las/os aspirantes debían encontrarse dentro de las medidas del "biotipo nacional" de Profesores de Educación Física de la estudiante Adelina Giordano en 1943 donde la misma hacía referencia explícita a los postulados biotipológicos, utilizándolos como marco para analizar las vinculaciones con la educación física?.

Otro de los espacios de gran importancia donde los postulados biotipológicos tuvieron cierta circulación previa a la incorporación en 1948 fue en los Congresos Panamericanos de Educación Física ${ }^{9}$. Los mismos tenían un peso significativo en la medida que su dinámica de funcionamiento implicaba la consagración de acuerdos entre los participantes sobre las políticas a implementarse vinculadas a la educación física estatal. Tanto en el primer congreso (dos Santos, 2012, pp. 185-186) desarrollado en Rio de Janeiro en 1943 como en el segundo desarrollado en México en 1946, la biotipología formó parte de los contenidos que se abordaron. Específicamente, dentro de este último, en la sección II, vinculada a "biología, medicina y ciencias aplicadas a la educación física", se mencionaba el desglosado siguiente: "10:.- Biotipología y Educación Física. 11:-Desviaciones de la normalidad morfológica y fisiológica. - Criterio de apreciación. - Papel de la Educación Física frente a estos problemas." (CNEF, 1946, s/n). Temáticas éstas sobre las cuales se discutió en el congreso llegando a la conclusión de que la educación física se debía basar en la biotipología para orientar científicamente sus prácticas:

Siendo la educación física, una actividad basada en las ciencias que conducen al conocimiento total del individuo, para orientar los impulsos vitales de sus fuerzas somáticas, fisiológicas, con propósitos comunes a la educación en todos los pueblos; y siendo éstos, cualquiera sea su origen o predominancia racial, presentan entre sus individuos las mismas variantes biotipológicas, en base a las cuales procede la orientación científica de la educación física. (CNEF, 1946: s/n).

Los referentes de la CNEF en el congreso hacían alusión a que la preocupación en torno a que la educación física se generara y dictara en base a grupos "homogéneos" estuvo presente desde el primer congreso panamericano. Esta preocupación la encontramos enunciada por diferentes actores locales desde los primeros años de la conformación de la CNEF y fue plasmada como política de la comisión (sobre todo a partir de la confección de las fichas antropométricas ${ }^{10}$ ) en el "primer plan de acción" de 1923. Desde la perspectiva de los representantes de la educación física regional, la homogeneidad de los grupos debía estructurarse en función de la constitución biológica de los estudiantes. Para ello, comenzaron a pensar y proyectar la utilización de herramientas que posibilitaran establecer esas cualidades homogéneas de la población y de los grupos específicos sobre los cuales desarrollar las prácticas de educación física. La antropometría y la biotipología, en tanto disciplinas encargadas de visibilizar los valores "normales" de los grupos y las cualidades individuales en relación a la media, fueron 
utilizadas como "ciencias" auxiliares y fundamentales para la labor de una educación física que pregonaba por la generación de cuerpos acorde a los valores "normales".

Por último, en relación a los antecedentes vinculados a los postulados biotipológicos, nos interesa visibilizar lo mencionado por Julio Pereyra en 1946 en ocasión de las "semblanzas de Jess Tess Hopkins". Pereyra ${ }^{11}$ (1946, p.15) hacía referencia a que la labor de Hopkins ${ }^{12}$ habría estado vinculada, entre otras cosas, a "la evolución del concepto educativo ontogenético y biotipológico afirmándose definitivamente la idea que considerar que el cuerpo y la mente forman una unidad indivisible". Los enunciados de Pereyra son de una significación importante en la medida que visibilizan el predominio dado a la ciencia iniciada por Pende y a las posibilidades de que Hopkins haya sido un continuador de los postulados biotipológicos. A su vez, emergen dos elementos más que nos resultan significativos: por un lado, la identificación con la ontogénesis que fue una de las principales políticas desarrolladas por el fascismo y de fuertes continuidades discursivas con la eugenesia. Por otro lado, el señalamiento de la biotipología como una disciplina que aunaba mente y cuerpo: elementos presentes dentro de los postulados religiosos de los cuales Pende y los referentes de la YMCA en Uruguay —como Hopkins - eran afines.

Entendemos que algunos de los hechos mencionados en este apartado nos permiten visualizar que los enunciados vinculados a la biotipología tuvieron cierta circulación previa a la incorporación definitiva de la asignatura y que los mismos pueden pensarse como una de sus condiciones de posibilidad para la emergencia de la misma dentro del curso de profesores de educación física. Desde nuestro punto de vista, la materialización más clara de los lazos establecidos entre la ciencia iniciada por Pende y la educación física en el Uruguay quedó configurada en ocasión del segundo "plan de estudios" con el que contó la formación de profesores de educación física en 1948. Postulamos que esta incorporación lejos de ser una ruptura discursiva con la matriz en torno a la cual se estaba confeccionando el campo de la educación física a nivel regional presenta grandes continuidades. La misma puede ser pensada como una tecnología auxiliar y solidaria con las lógicas biopolíticas (Foucualt, 2009) tendientes a reducir los sujetos a meros organismos biológicos y funcionales a la economía política.

\section{La incorporación al plan de estudio}

En ocasión de la elaboración del segundo plan con el que contó la formación de profesores de educación física en el Uruguay, se incorporó en el tercer y último año de la carrera la asignatura denominada "biotipología", perdurando en la currícula hasta $1965^{13}$. La asignatura, desde su comienzo, y por lo pronto hasta su finalización, estuvo a cargo del Dr. Francisco Devincenzi, encontrándose en el primer trimestre del tercer año de la carrera y con una carga horaria total de 27hs. Para este trabajo tomanos 4 tesinas de egreso $^{14}$ de estudiantes que, en su época (1943-1954), optaron por vincular sus trabajos a las ideas biotipológicas. Particularmente, nos centraremos en visibilizar algunas de las articulaciones generadas entre educación física, biotipología y eugenesia a partir de los enunciados encontrados en las tesis de las/os estudiantes. En términos generales, para el desarrollo de los conceptos sobre la biotipología, en los cuatro trabajos las/os estudiantes recurrieron, sobre todo, a los postulados de Nicola Pende y en menor medida aparecen referencias a Ernst Kretschmer, Waldemar Bernardinelli, Gregorio Marañón (también 
referentes del área de la biotipología) y los apuntes de clase sobre las disertaciones de Francisco Devincenzi.

\section{Biotipología y educación física: el horizonte utópico eugenésico}

En relación a la formación de profesores de educación física en el Uruguay, se podría pensar que biotipología y educación física mantuvieron un doble entrecruzamiento vinculados a los objetivos macro de ambas disciplinas. Por una parte, la biotipología le brindo elementos conceptuales y procedimentales para efectuar de una "mejor manera" la educación del cuerpo de los individuos. El profesor contaba a partir de los postulados de la biotipologia con una mayor cantidad de conocimientos vinculados a las cualidades corporales de cada individuo —donde se incluían aspectos: morfológicos; temperamentales; morales; y psicológicos - y, por otro lado; con la información relativa a las prácticas que debía desarrollar — acorde a esa individualidad- para propiciar su mejor rendimiento biotipológico. En la línea de la tradición del campo de la educación física (Dogliotti, 2012, Rodríguez, Giménez, 2012), se puede pensar que estos conocimientos aportados desde la biotipología a las/os docentes se encontraban más próximos a un conjunto de mecanismos tendientes a la identificación y prescripción de prácticas sobre los cuerpos de las/os estudiantes que a un conocimiento a ser enseñado. Los postulados biotipológicos le brindaron al/la docente una mayor cantidad de elementos para establecer y justificar las "mejores" formas de encauzar al cuerpo dentro de su "normalidad biotipológica". La enseñanza no estaba pensada en tanto un conocimiento al que todas/os podían acceder sino que la predominancia era de la determinación biotipológica - por parte de la/el docente- de las mejores cualidades de los individuos para el tipo de práctica. Los postulados biotipológicos servían como herramientas para la selección y eliminación de los individuos a las diferentes prácticas a desarrollar: una de las ideas claves a partir de la cual se articula en torno al discurso eugenésico. Por otra parte, la educación física era visualizada como una disciplina aliada de la biotipología en la medida que se entendía que colaboraba con el desarrollo del ambiente propicio para el fortalecimiento de la raza y, a su vez, desarrollando prácticas de fortalecimiento de las cualidades específicas de cada biotipo, propiciaba la eficiencia del "organicismo social".

En la medida que el discurso de la "eugenesia latina"15 puede ser pensado como un conjunto de prescripciones tendientes al perfeccionamiento de la raza por medio del desarrollo físico, moral e intelectual de los individuos, donde convergeran mecanismos de selección y depuración de los individuos que no colaboran con ese desarrollo racial, la biotipología y la educación física articuladas, como se visibiliza en el caso del curso de profesores de educación física de la CNEF, establecieron -en conjunto y por separadofuertes lazos de solidaridad con ese ideal eugenésico, colaborando en su tarea. Una de las manifestaciones más significativas sobre esta articulación la encontraremos en uno de los trabajos de las/os estudiantes:

El problema de las desviaciones morfológicas no es entonces puramente un problema estético, ni un problema de salud y de longevidad, es también un problema espiritual y un problema moral [...] Surge así un problema de eugenia, de perfeccionamiento de la raza, por los medios adecuados al desarrollo físico perfecto mediante un funcionamiento glandular normal. La humanidad moderna debe buscar y encontrar en una nueva vida física, el vigor que fue la herencia de la antigüedad y que hemos 
perdido por múltiples factores inherentes al trajín de la vida misma. (Giordano, 1943, p. 43).

Emergen en estos enunciados algunos de los elementos centrales mediantes los cuales biotipología, eugenesia y educación física convergieron en un "horizonte utópico" (Sapriza, 2001): el "perfeccionamiento de la raza". La educación física, asumiendo los postulados biotipológicos que establecían pautas de normalidad -vinculadas tanto a lo espiritual, moral, como morfológico- debía propiciar los "medios adecuados" para el desarrollo del "físico perfecto". El físico, lo morfológico se constituyó como uno de los elementos claves en la consagración de la armonía biotipológica:

Cada desarmonia morfológica es la consecuencia de una desarmonía funcional. En consecuencia: si las desarmonías de las formas dependen de una desarmonía de la función, para corregir la desarmonía morfológica hay que corregir la desarmonia funcional. Si estudiamos, entonces, la morfología humana a través de las distintas etapas de la historia, podremos ver reflejadas en la línea de sus formas el funcionamiento glandular. El individuo, dice Giovanni, no es otra cosa que el resumen de sus ancestrales y del medio ambiente. [...] El ejercicio físico, considerado dentro del factor ambiental, actúa a través de las influencias hormonales y él debe tender en la hora actual a la perfección de la salud que es la belleza de la función. (Giordano, 1943, p. 13).

Mediante su desarrollo, se entendía que se podía incidir favorablemente en los elementos endocrinos, regulándoles y ésto traería aparejado la mejora y el equilibro con el resto de las caras de la pirámide que conforman al biotipo. A su vez, se recurre a la idea de una herencia perdida, no favorecida por las prácticas ambientales que no posibilitan que esas cualidades que han formado parte de la raza y que perduran en las generaciones por medio de la transmisión hereditaria se haga presente. La educación física debía actuar favoreciendo que esa herencia pueda emerger nuevamente.

Por otra parte, en clara sintonía con los postulados eugenésicos y con las lógicas de los sistemas totalitarios (Rodríguez, Giménez, 2016), se establece la idea de que existe un ideal de perfección, que existe una imagen, una verdad, que representa el ideal al que todos deben aspirar a emparentarse. En este entramado, el biotipo "verdaderamente" armónico —que no solo incluía parámetros físicos sino también morales, intelectivos y espirituales- se convirtió en el "nuevo" modelo a partir del cual la educación física debía orientar sus prácticas para alcanzarlo:

La existencia de una tal armonía en grado suficiente es la condición sin la cual no es posible clasificar al sujeto como verdaderamente robusto en el sentido biotipológico. En el caso que uno de los lados del biotipo sea defectuoso por exceso o defecto, se considera que el sujeto es desarmónico y un falso robusto aunque puede ser fuerte de cuerpo 0 de inteligencia. Esta distinción de verdadera robustez armónica 0 disarmónica es de interés para la aplicación práctica médica y educativa del examen biotipológico. La biotipología tiene pues como finalidad inmediata, hacer un reajuste nuevo para justipreciar líneas de conducta...(Giordano, 1943, pp. 22-23)

Las prácticas de educación física a ser desarrolladas sobre los cuerpos de la población debían basarse en una de las herramientas claves de la biotipología como fueron los exámenes. La aspiración debía colocarse en alcanzar la perfección del individuo, el verdadero "robusto", una raza perfecta. Los postulados biotipologicos por 
medio de las prácticas de la educación física encontraron una vía de gran importancia mediante la cual "hacer carne" sus conceptos, disciplinar el cuerpo, moldearlo en función de la obtención de un "ideal do Eu" (Rodríguez, Giménez, 2016). En términos de Rodriguez (2016):

quando tudo tem que se parecer com o ideal do Eu que funciona na conformação de um grupo, é possível que se ponha a funcionar uma espécie de sistema paranoico (ADORNO, 2008), sistema que está na base do fascismo, da negação do outro. [...] Uma política baseada na semelhança é, finalmente, fascista. (pp. 52, 165)

Biotipología y educación física se aunaron en la reducción de los sujetos a un mero organismo a ser gobernados por los valores de una nueva disciplina de la normalidad que tuvo como objetivo macro un ideal eugenésico: producir, a imagen y semejanza, la raza perfecta.

\section{Conclusiones}

En el recorrido de este trabajo buscamos visibilizar y analizar las formas en las que educación física y la biotipología se articularon en torno a la formación de profesores de educación física en el Uruguay. Dentro del recorrido identificamos que desde la formulación de la biotipología en Italia por el médico Nicola Pende la educación física fue identificada como una importante herramienta para la concreción de los objetivos que se buscaban desarrollar desde los postulados biotipológicos. Estos últimos, fueron visualizados como importantes insumos para la prescripción de los deportes específicos que debía desarrollar cada individuo, acorde a sus cualidades biotipológicas y bajo la idea de fortalecer el "organismo social". Este tipo de articulaciones quedaron puestas de manifiesto en una de las instituciones más importantes en América del Sur vinculadas al desarrollo de la biotipología y la eugenesia como fue la Asociación Argentina de Biotipología Eugenesia y Medicina Social. A su vez, tanto en Argentina como en Brasil, señalámos que los supuestos teóricos- metodológicos de la biotipología tuvieron una incidencia considerable en la conformación del campo de la educación física, generando importantes afectaciones para la educación del cuerpo.

En relación al campo de la educación física en Uruguay, identificamos que los postulados de la biotipologia tuvieron una circulación previa a su incorporación definitiva al plan de estudio de 1948. Postulamos que estas referencias pueden ser pensadas, en un escenario amplio, como algunas de las condiciones que posibilitaron el ingreso de la biotipología al campo de la educación física. Resulta significativo señalar que esta asignatura formó parte de la formación de profesores de educación física en Uruguay -por lo menos durante veinte años- pudiendo haber generado importantes afectaciones para la constitución disciplinar del campo. Aún así, mencionamos que los postulados biotipológicos no representaron una ruptura en torno a la matriz epistémica -vinculada a la ciencias biomédicas- sobre la que se edificó el campo de la educación física en Uruguay y la región, sino más bien que los mismos mantienen fuertes continuidades discursivas con la misma.

En relación a los trabajos realizados por los estudiantes vinculados a la asignatura biotipología, se puede identificar que el cuerpo fue significado, sobre todo, en tanto un 
organismo a ser regulado por los valores de las ciencias biomédicas. La centralidad de los conocimientos vertidos por la biotipología estuvieron en la determinación del justo deporte para cada individuo y en el desarrollo de esa práctica sobre el cuerpo del/la estudiante y no, por ejemplo, en una vinculación con el conocimiento de los diferentes deportes que forman parte de la cultura.

La enseñanza quedaba estrechamente vinculada a las cualidades biotipológicas donde a cada individuo le correspondía "la justa" educación que su biotipo demandaba, las características biotipológicas eran las que estipulan el tipo de enseñanza que se debía impartir sobre los cuerpos. En este sentido, se puede inferir que los conocimientos aportados desde la biotipología le brindaron mayores insumos a las/os profesoras/es para la tarea de encauzar al cuerpo de la población en función de postulados que buscaban favorecer la mayor eficiencia del individuo en tanto energía para el desarrollo de un Estado-organismo. Dentro de esta lógica, la educación física cobró una importancia relevante en la medida que era visualizada y producida como una potente herramienta para garantizar el óptimo funcionamiento del "organicismo social". Eugenesia, biotipología y educación física convergieron en un "horizonte útopico" vinculado al perfeccionamiento y evolución de la raza por medio de su desarrollo biológico generando importantes afectaciones para la educación del cuerpo en el Uruguay.

\section{Fuentes}

CNEF (1946). Il Congreso Panamericano de Educación Física. 1ero al 15 de octubre de 1946. Informe de la delegación oficial. CNEF, Montevideo, junio, págs. s/n.

Galton, F. (1909). Essays in eugenics. London, United Kingdom: The eugenics education society

Giordano, A. (1943). Glandulas de secreción interna y biotipologia en la educación física. (Tesis de grado). Curso para Profesores de Educación Física, CNEF. Montevideo. págs. $1-72$.

Pende, N. (1933). Biotipología y educación física. Anales de Biotipología, Eugenesia y Medicina Social, Argentina, núm. 8, pp. S/n.

Pereyra, J.(1946). Semblanza del Sr. Jess T. Hopkins. Montevideo: Centro Cultural de Profesores de Educación Física.

\section{Bibliografía}

Bashford, A., \& Levine, P. (Eds.). (2010). The Oxford Handbook of the History of Eugenics. New York, Estados Unidos: Oxford University Press

Dogliotti, P. (2012). Cuerpo y currículum: discursividades en torno a la formación de docentes de educación física en Uruguay (1874-1948). (Tesis de Maestría). Enseñanza Universitaria, Comisión Sectorial de Enseñanza, Área Social, Universidad de la República, Montevideo, Uruguay. 
Dos santos Silva, A. (2012). Nos dominios do corpo e da espécie: Eugenia e biotipologia na constituição disciplinar da educacão Fisica. (Tesis doctoral). Programa de PósGraduação em Ciências do Movimento Humano, Universidade Federal do Rio Grande do Sul, Brasil.

Foucault, M. (2009). Historia de la sexualidad. La voluntad del saber. Buenos Aires, Argentina: Siglo XXI.

Galak, E. (2014). Educación del cuerpo y política: concepciones de raza, higienismo y eugenesia en la Educación Física Argentina. Movimento, Porto Alegre, V. 20, núm. 4, pp. 1543- 1562.

Galak, E. (2016). Educar los cuerpos al servicio de la política. Cultura física, higienismo, raza y eugenesia en Argentina y Brasil. Buenos Aires, Argentina: Biblos.

Galera, A. (2005). Hacia una fisiología del delito: el modelo biotipológico de Nicola Pende. En Miranda, M. y Vallejo, G. (Eds.), Darwinismo social y eugenesia en el mundo latino (pp. 363 - 374). Buenos Aires, Argentina: Siglo XXI.

Miranda, M., y Vallejo, G. (Eds.). (2005). Darwinismo social y eugenesia en el mundo latino. Buenos Aires, Argentina: Siglo XXI.

Miranda, M., y Vallejo, G. (Eds.). (2012). Una historia de la eugenesia: Argentina y las redes biopolíticas internacionales 1912-1945. Buenos Aires, Argentina: Biblos.

Ramacciotti, K. (2005). Las huellas eugénicas en la política sanitaria argentina (19461955). En Miranda, M., y Vallejo, G. (Eds.), Darwinismo social y eugenesia en el mundo latino (pp. 311- 350). Buenos Aires, Argentina: Siglo XXI.

Reggiani, A. (2005). La ecología institucional de la eugenesia: repensando las relaciones

entre biomedicina y política en la argentina de entreguerras. En Miranda, M., y Vallejo, G. (Eds.), Darwinismo social y eugenesia en el mundo latino (pp. 273-310). Buenos Aires, Argentina: Siglo XXI.

Reggiani, A. (2014). Eugenesia y cultura física. Tres trayectorias históricas: Francia, Gran Bretaña y Argentina. En Scharagrodsky, P. (Ed.), Miradas médicas sobre la cultura física en Argentina 1880-1970 (pp.17 - 58). Buenos Aires, Argentina: Prometeo.

Rodríguez, Antúnez, C. (2016). El examen de ingreso al Curso de Profesores de Educación Física en el Uruguay. Una herramienta de selección en clave biotipológica. Cartografías del Sur, Revista Multidisciplinaria en Ciencia, Arte y Tecnología de la Universidad Nacional de Avellaneda. Argentina. № 4, octubre, pp. 196 - 223.

Rodríguez, Giménez, R. (2012). Saber del cuerpo: una exploración entre normalismo y universidad en ocasión de la educación física (Uruguay, 1876-1939). (Tesis de Maestría). Enseñanza Universitaria, Comisión Sectorial de Enseñanza, Área Social, Universidad de la República, Montevideo, Uruguay.

Rodriguez, Gimenez, R. (2016). Saber do corpo: entre o político e a política. (Tesis doctoral) Programa de PósGraduación Interdisciplinar en Ciencias Humanas de la Universidad Federal de Santa Catarina, Florianopolis, Brasil.

Teja, Á. (2002). Deporte y relaciones internacionales durante el fascismo en Italia. En González, Teresa (Ed.), Sport y autoritarismos. La utilización del deporte por el comunismo y el fascismo (pp. 241-280). Madrid, España: Alianza. 
Torrón, A. (2015). Gimnasia y Deporte en el Instituto Superior de Educación Física (19391973): su configuración y su enseñanza. (Tesis de Maestría). Enseñanza Universitaria, Comisión Sectorial de Enseñanza, Área Social, Universidad de la República, Montevideo, Uruguay.

Vallejo, G. (2005). Las formas del organicismo social en la eugenesia latina. En Miranda, M., y Vallejo, G. (Eds), Darwinismo social y eugenesia en el mundo latino (pp. 231- 272). Buenos Aires, Argentina: Siglo XXI.

Vallejo, G. (2012). Roma: capital de un Imperio bajo el signo de la biología política (19361942). Dynamis, Madrid, 32 (1), pp. 115-140.

Vallejo, G. (2018). La hora cero de la eugenesia en la Argentina: disputas e ideologías en el surgimiento de un campo científico, 1916-1932. História, Ciências, Saúde Manguinhos, Rio de Janeiro, v.25, supl., ago, pp.15-32.

Sapriza, G. (2001). La "utopía eugenista" Raza, sexo y género en las políticas de población en el Uruguay (1920- 1945). (Tesis de Maestría, inédita). Facultad de Humanidades y Ciencias de la Educación-UdelaR, Montevideo, Uruguay.

\section{Notas}

${ }^{1}$ La investigación es el resultado de una tesis de posgrado desarrollada en la Maestria en Enseñanza Universitaria de la Comisión Sectorial de Enseñanza y la Facultad de Humanidades y Ciencias de la Educación- UdelaR, Uruguay. Para el desarrollo de la misma se obtuvo la beca otorgada por la CAP-UdelaR y la financiación del ISEF-UdelaR por medio del cargo docente centrado en la investigación.

2 Pende, (1922): "La debolezze di costituzione".

${ }^{3}$ La eugenesia fue una disciplina iniciada por Francis Galton (1822-1911) en Reino Unido sobre fines de siglo XIX. En términos generales, Galton se propuso crear una ciencia que indagara sobre las cualidades biológicas hereditarias de los seres humanos "eminentes", los que poseían el "genio" y, a su vez, dar cuenta de las condiciones biológicas hereditarias de aquellos que no lo poseían. Esta información, siguiendo lo estipulado por el autor (Galton, 1909), debía servir como un insumo para que el Estado trazara políticas tendientes a que solo se reproduzcan los "eminentes", favoreciendo de este modo la "superviviencia de los más aptos" y con ello propiciar la "evolución racial". Para una mayor profundización sobre las implicancias de la eugenesia en el escenario internacional ver: Bashford y Levine (2010). Para el contexto regional ver: Miranda y Vallejo (2005, 2012).

${ }^{4}$ Enrique Romero Brest fue un actor clave en la consolidación de la enseñanza de la Educación Física en Argentina, fundando el primer Instituto Superior de Educación Física encargado de la formación de profesores en el área.

${ }^{5}$ En 1939, por decreto de ley, se creó el curso para profesores de educación física. La creación del curso puede pensarse como un hito en cuanto a la formación de un personal especializado en educación física en el país ya que era el único dentro de la órbita pública- estatal y que se encontraba a cargo de la CNEF. Para una mayor profundización sobre las implicancias de este conformación ver: Dogliotti (2012), Rodríguez, Giménez (2012), Torrón (2017).

6 Dentro de las disposiciones generales que se estipularon para poder ingresar a la formación de tres años - y que habilitaba para trabajar en la órbita estatal vinculada a las políticas de la CNEF- se confeccionaron un conjunto de pautas vinculadas a aspectos morales, físicos e intelectuales que las/os aspirantes debían superar en caso de querer acceder a la formación.

7 Para una mayor profundización sobre este punto ver: Rodríguez, Antúnez (2016).

8 Adelina Giordano (1943) "Glándulas de secreción interna y biotipología en la Educación Física". Partiendo de algunas premisas vinculadas al catolicismo y siendo, desde nuestro punto de vista, uno de los trabajos de mayor "rigurosidad" sobre los postulados de la endocrinología de Pende, la autora se centró en demostrar cómo los factores endócrinos jugaban un papel de suma importancia en el desarrollo psicológico del individuo y como las características biológicas determinan el tipo de práctica que se debía desarrollar.

9 Los congresos panamericanos de educación física surgieron como una iniciativa Raul B. Blanco por 1938 (Dogliotti, 2012). Blanco había sido formado en la escuela de lideres de la YMCA de Montevideo y ocupó diferentes espacios de importancia dentro de la Comisión Nacional de Educación Física de Uruguay. En 
1941, con el objetivo de propiciar el primer congreso panamericano, Blanco fundó el Instituto Panamericano de Educación Física con sede en Estados Unidos (Dogliotti, 2012, p. 173). En ese año también fue nombrado como "secretario provisorio" para propiciar la organización del primer congreso a desarrollarse en Rio de Janeiro- Brasil en julio de 1943. El siguiente de estos congresos fue en México y el tercero en Montevideo en 1950. Los congresos pueden ser pensados como espacios significativos en cuanto a la circulación y producción de los diferentes discursos en torno a los cuales se estaba desarrollando el campo de la educación física. Tanto los enunciados vinculados a la eugenesia como a la biotipología tuvieron su lugar específico dentro de estos espacios y fueron abordados por los referentes del campo. A su vez, la dinámica de los mismos implicaba, además de los trabajos en comisiones, la visibilización de los acuerdos alcanzados por las/os participantes. Estas resoluciones tenían un peso relevante en cuanto a la implementación de políticas dentro del área específica, siendo espacios donde el desarrollo académico quedaba aunado de forma significativa al desarrollo de políticas públicas y estatales.

16 En las décadas de 1920 y 1930 en Argentina, Brasil y Uruguay comenzaron a instalarse discursos tendientes a sostener la implementación de las fichas antropométricas en diferentes niveles pero, sobre todo, en el contexto escolar (Galak 2014, Dos Santos 2012, Miranda y Vallejo 2005, Reggiani 2005, Ramacciotti, 2005). El campo de la educación física no fue ajeno a este proceso, colaborando de forma efectiva en su implementación.

${ }^{11}$ Julio Pereyra se formó en e Springfield College de la YMCA- Massachussets- EE.UU, egresando de esta institución en 1923. Dentro de la CNEF se desempeñó en diferentes cargos dentro de los que se desataca su labor dentro del curso para profesores de educación física de la CNEF.

12 Jess Tess Hopkins, fue uno de los principales referentes discursivos en la consolidación de la educación física en el Uruguay. Para una mayor profundización sobre la figura de Hopkins en la conformación del campo de la educación física en el Uruguay ver: Dogliotti (2012).

${ }^{13}$ En 1966, se elaboró el cuarto plan de estudio del curso de profesores de educación física donde la disciplina, por razones que aún no hemos identificado; fue retirada de la estructura curricular.

${ }^{14}$ Los títulos de las tesis son los siguientes: Adelina Giordano (1943) "Glándulas de secreción interna y biotipología en la Educación Física"; Susana Mangini (1950) "El deporte y la biotipología racial"; Marcos Feldman (1951) "Educación Física en el Obrero"; Dina Achenbach (1954) "Psicología del niño y la educación física".

${ }^{15}$ En la linea de lo propuesto por Vallejo (2018: 29) puede pensarse como una "expresión de un saber integrador de ciencia, política y religión, donde, bajo una fuerte impronta ambiental, eran repelidos anteriores cuestionamientos al papel de la Iglesia, para quedar despejado el camino hacia el ejercicio de una coerción disimulada". 